\title{
Graft tunnel placement and orientation are key determinants of internal knee mechanics after ACL reconstruction
}

Michael F. Vignos

Colin R. Smith

Joshua D. Roth

Jarred M. Kaiser

Geoffrey S. Baer

Richard Kijowski

Darryl G. Thelen

\section{Video Abstract}

Keywords: American Journal of Sports Medicine, biomechanics, ACL reconstruction, osteoarthritis, anatomic graft placement, graft tunnel, graft angle, tibiofemoral kinematics, computational model, simulation, joint mechanics, MRI, laxity, compliance index, tibial translation, graft stiffness, tibial plateau, ACL loading, internal rotation, cartilage contact

Posted Date: February 26th, 2021

DOI: https://doi.org/10.21203/rs.3.rs-279566/v1

License: (c) (i) This work is licensed under a Creative Commons Attribution 4.0 International License. Read Full License 


\section{Abstract}

Early-onset knee osteoarthritis is commonly observed after ACL reconstruction, and may be attributable to residual abnormal joint mechanics following surgery. Graft tunnel placement is a surgical factor that may affect internal knee joint mechanics, and thereby joint health. In this study, researchers from the University of Wisconsin used a combination of MRI and computational modeling to assess the consequences of graft placement on the loading of cartilage in the knee. The findings, reported in the _American Journal of Sports Medicine_, highlight the aspects of graft placement that surgeons should most closely consider to restore normal mechanics during ACL reconstruction. MRI measurements were performed to assess the ACL tunnel location and graft angle in the knees of 18 participants who had undergone ACL reconstruction. A clinical laxity test was performed on each subject following the completion of post-surgery physical therapy. Dynamic MRI was used to measure tibiofemoral kinematics and cartilage contact during active flexing and extending of the knee. The team also constructed 500 virtual ACL reconstruction models based on a previously validated computational model of the knee. The models captured a range of different ACL tunnel locations, graft stiffness, and initial tension-and they were used to simulate laxity tests, active knee extension, and walking. Both the MRI measurements and simulations revealed the same pattern: a more vertical graft in the sagittal plane was significantly related to greater knee laxity, greater anterior tibial translation, and greater internal tibial rotation during active knee extension. These effects carried over to the simulations of walking. In addition to allowing for greater anterior tibial translation, a more vertical ACL graft led to greater ACL loading and a posterior migration of contact on the tibial plateaus. Overall, the study provides clinical and biomechanical evidence that functional knee mechanics after ACL reconstruction are sensitive to graft tunnel location. In particular, variations in post-operative cartilage loading was most sensitive to the orientation of the graft in the sagittal plane. This link between graft placement and cartilage loading may have implications for understanding the elevated risk of osteoarthritis observed following ACL reconstruction. 